\title{
Checklist da classe appendicularia (Chordata: Tunicata) do Estado de São Paulo, Brasil
}

\author{
Luz Amelia Vega-Pérez ${ }^{1,3}$, Meiri Aparecida Gurgel de Campos $^{2}$ \& Katya Patrícia Schinke ${ }^{1}$
}

\author{
${ }^{1}$ Departamento de Oceanografia Biológica, Instituto Oceanográfico, Universidade de São Paulo - USP, \\ Praça do Oceanográfico, n. 191, Butantã, CEP 05508-120, São Paulo, SP, Brasil \\ ${ }^{2}$ Centro de Ciências Naturais e Humanas, Universidade Federal do ABC - UFABC, \\ Rua Santa Adélia, n. 166, Bairro Bangu, CEP 09-210-170, Santo André, SP, Brasil \\ ${ }^{3}$ Autor para correspondência: Luz,Amelia Vega-Pérez, e-mail: lavega@usp.br
}

\begin{abstract}
VEGA-PÉREZ, L.M., CAMPOS, M.A.G. \& SCHINKE, K.P. Checklist of class appendicularia (Chordata: Tunicata) from São Paulo State, Brazil. Biota Neotrop., 11(1a): http://www.biotaneotropica.org.br/v11n1a/en/ abstract?inventory+bn0401101a2011.
\end{abstract}

\begin{abstract}
The appendicularians are planktonic tunicates exclusively marine, characterized by the presence of the notochord in the adult stage and the lack of the peribranchial cavity and cloacae. The body is transparent and divided into two regions: a trunk, exceptionally exceeding $5 \mathrm{~mm}$, and a tail which is generally several times longer than the trunk. These organisms, with exception of Oikopleura dioica, are hermaphroditic protandric, and release their gametes directly to the water column. They have both very high fecundity and growth rate. The primarily food item of appendicularians is pico-nannoplankton, virus, bacteria and mucoid substances. They feed by means of a complex mucous structure, the "house" which is secreted by the trunk and used as tangential flow filter to concentrate ambient food particles prior to their collection by the pharyngeal filter. The appendicularians are important food item of others components of the zooplankton, including larval and adult fish. On the other hand, the marine snow produced by them contribute substantially to the carbon turn over time. The sinking rates of their faecal pellets and discharged houses can reach deep waters and their repackaging activity may play a significant role in channeling short lived carbon toward sequestration. The Appendicularians are conspicuous members of marine zooplankton, occurring in both neritic and oceanic regions of all oceans. High number of species is epipelagic, being most abundant around 100-200 m. However, some species inhabit the meso-and bathypelagic regions. The highest diversity of Appendicularians has been reported from warmest waters. At the moment 82 species belonging to the Oikopleuridae, Fritillariidae and Kowalesvkiidae families were identified, and from these, 43 species have been recorded in South Atlantic and 29 species in Brazilian waters. In São Paulo State twenty species were found along the coastal waters.
\end{abstract}

Keywords: appendicularia, biodiversity of the State of São Paulo, BIOTA/FAPESP Program.

Number of species: in the world: 82, in Brazil: 29, estimated in São Paulo State: 29.

VEGA-PÉREZ, L.M. CAMPOS, M.A.G. \& SCHINKE, K.P. Checklist da classe appendicularia (Chordata: Tunicata) do Estado de São Paulo, Brasil. Biota Neotrop., 11(1a): http://www.biotaneotropica.org.br/v11n1a/ pt/abstract?inventory+bn0401101a2011.

Resumo: Os tunicados da classe Appendicularia são organismos pelágicos, exclusivamente marinhos, caracterizados pela ausência da cavidade branquial e da cloaca, bem como pela retenção de notocorda e cauda muscular no estágio adulto. O corpo, delicado e transparente, é formado pelo tronco que raramente excede os $5 \mathrm{~mm}$ e pela cauda, várias vezes mais longa que o tronco. São hermafroditas protândricos, exceção feita à espécie Oikopleura dioica, que na época da reprodução lançam os gametas na água onde ocorre a fecundação. Os apendiculários se alimentam basicamente de pico-nanoplâncton, bem como de pequenas diatomáceas, vírus, bactérias e material coloidal. Durante a alimentação utilizam a casa ou "house", secretada pelo epitélio glandular do tronco, que possui filtros internos para concentrar as partículas antes de serem selecionadas e ingeridas. $\mathrm{Na}$ teia alimentar marina, são elo importante entre o pico-nanoplâncton e os níveis tróficos superiores já que servem de alimento para os outros componentes do zooplâncton, incluindo peixes de interesse comercial. Sua elevada taxa de fecundidade e de crescimento, aliada à capacidade de produzir quantidades significativas de matéria orgânica representada pela casas descartadas e pelotas fecais, os tornam peça fundamental no fluxo de energia. A neve marinha produzida por estes organismos, representa uma importante fonte de carbono para as camadas mais profundas. Os apendiculários são encontrados em todas as regiões neríticas e oceânicas do mundo, sendo mais abundantes na camada dos 100 a 200 m, embora algumas espécies habitem as regiões meso e batipelágicas. A maior diversidade de espécies foi registrada em águas quentes. Das 82 espécies identificadas até o momento em todo o mundo, 43 foram citadas para o Oceano Atlântico Sul, 29 para as águas brasileiras e 20 espéies para o Estado de São Paulo.

Palavras-chave: appendicularia, biota paulista, Programa BIOTA/FAPESP.

Número de espécies: no mundo: 82, no Brasil: 29, estimadas no Estado de São Paulo: 29. 


\section{Introdução}

A classe Appendicularia é constituída de espécies exclusivamente marinhas, cujo nome deve-se à presença do apêndice propulsor denominado cauda. São conhecidos também como Larvacea devido à sua semelhança com as larvas dos outros Tunicata (Gorsky \& Palazzoli 1989). Esses organismos são um dos componentes mais frequentes do zooplâncton, embora raramente sejam dominantes em termos de biomassa (Hopcroft \& Roff 1995, Choe \& Deibel 2008).

A classe é constituída por 82 espécies pertencentes às famílias Oikopleuridae Lohmann, 1896; Fritillariidae Seeliger, 1895 e Kowalevskiidae Lahille, 1888; que se diferenciam pelos formatos do tronco, endóstilo, espiráculos, parede do estômago, epitélio oicoplástico, gônadas e cauda. Dessas três famílias, Oikopleuridae é a que possui o maior número de gêneros e espécies descritas (Esnal 1977, 1981, 1999, Fenaux et al. 1998).

As principais características que distinguem os apendiculários dos outros Tunicata são: a ausência da cavidade branquial e da cloaca, a presença de epitélio glandular que reveste a parte anterior do tronco, bem como a retenção de notocorda e cauda muscular no estágio adulto (Fenaux 1977).

Os apendiculários são organismos solitários, de tamanho pequeno, aspecto delicado, transparentes e luminescentes. O corpo é formado pelo tronco e pela cauda, várias vezes mais longa que o tronco. Este último, que raramente ultrapassa os $5 \mathrm{~mm}$ de comprimento, é dividido em três regiões: faringo-branquial, digestiva e genital. São hermafroditas protândricos, exceção feita à espécie Oikopleura dioica que apresenta sexos separados. Na época da reprodução formam densos agregados e todos os indivíduos maduros lançam, ao mesmo tempo, os gametas na água onde ocorre a fertilização (Berril 1950, Last 1972, Esnal 1999, Brusca \& Brusca 2007).

Esses organismos são herbívoros e um dos poucos metazoários marinhos capazes de filtrar eficientemente as menores partículas presentes na coluna de água, incluindo bactérias (King et al. 1980, Deibel 1988, Capitanio et al. 1997, Hopcroft et al. 1998). Para obter o alimento utilizam a casa ou "house", considerada uma das mais complexas estruturas externas construídas por um organismo, que é secretada pelo epitélio glandular e cujo comprimento pode variar de $6 \mathrm{~mm}$ a $2 \mathrm{~m}$ aproximadamente (Alldredge 1977, Barham 1979, Dybas 1993). Durante a alimentação, o batimento da cauda orienta a água em direção aos filtros, existentes dentro da casa, onde as partículas são concentradas antes de serem selecionadas e ingeridas (Flood 1991, Alldredge \& Madin 1982, Flood et al. 1992). Esses filtros retêm partículas de até $0,1 \mu \mathrm{m}$, como cocolitoforídeos, flagelados nus, pequenas diatomáceas, dinoflagelados, bactérias e material coloidal, que são os principais itens alimentares dos apendiculários (Flood 1978, 2003, King et al. 1980, Hopcroft et al. 1998, Bone et al. 2003).

O processo de filtração intensa e contínua provoca periodicamente a obstrução dos filtros, o que exige o descarte da casa e a produção de uma nova a cada 2 ou 4 horas. Porém, dependendo da espécie, da disponibilidade de alimento, da taxa de alimentação e de fatores como a temperatura e salinidade, um indivíduo pode produzir entre 6 e 16 casas por dia (Esnal 1981, 1986, Sato et al. 2001, 2003). As casas descartadas pelos apendiculários são de extrema importância na cadeia trófica marinha, já que concentram grandes quantidades de fitoplâncton, muco e detrito no seu interior. Constituem agregados macroscópicos importantes no fluxo de carbono orgânico particulado e fontes ricas em alimento para algumas espécies que habitam as regiões mais profundas dos oceanos (Alldredge 1976a, 2005, Davoll \& Youngbluth 1990, Urban et al. 1992, Tomita et al. 1999, Maar et al. 2004, Dagg \& Brown 2005, Koski et al. 2007).
O ritmo de filtração intenso permite também que nas áreas onde os apendiculários são abundantes, filtrem de 30 a 60\% da água circundante em até 24 horas, concentrando milhões de células do fitoplâncton, mesmo em regiões oligotróficas (Alldredge 1977, Flood et al. 1992, Esnal 1996). Com isso, podem reduzir drasticamente a biomassa fitoplanctônica, regular o tamanho da população e sua disponibilidade para outros herbívoros (Barnes \& Hughes 1982, Esnal 1986, López-Urrutia et al. 2005, Sato et al. 2008). Porém, em ambientes costeiros e estuarinos esse mecanismo de alimentação pode contribuir para o aumento da transparência da água e a penetração de luz, estimulando o desenvolvimento do fitoplâncton (Dagg et al. 1996, Fernández \& Acuña 2003).

Na teia alimentar marinha, ainda, são elo importante entre o piconanoplâncton e os níveis tróficos superiores uma vez que fazem parte da dieta de peixes, muitos de interesse comercial (Last 1980, Ikewaki \& Tanaka 1993, Capitanio et al. 1997, Kurtz 1999), bem como de medusas, ctenóforos e quetógnatos (Alldredge 1976b, Uye \& Ichino 1995, Vega-Pérez \& Liang 1992, Liang \& Vega-Pérez 1995, 2001, 2002, Purcell et al. 2005, Urban-Rich et al. 2006). Por outro lado, seu ciclo de vida curto e elevada taxa de crescimento em condições favoráveis, permitem que atinjam picos de abundância rapidamente (Alldredge \& Madin 1982, King 1982, Hopcroft \& Roff 1995, Hopcroft et al. 1998, López-Urrutia et al. 2003). Essa característica aliada à capacidade de produzir quantidades significativas de matéria orgânica, representada pela casas descartadas e pelotas fecais (Taguchi 1982, Cima et al. 2002, Vargas et al. 2002), os tornam peça fundamental no fluxo de energia no ambiente marinho (Gorsky \& Fenaux 2003, Hopcroft \& Roff 1998, Deibel \& Paffenhöfer 2009). Isso porque a pelota fecal, além de refletir o tipo de fitoplâncton presente na área, serve como um eficiente meio de transporte de nutrientes, poluentes e outras partículas presentes na zona eufótica para as camadas mais profundas (Wefer et al. 1988, Urban et al. 1992, 1993).

Algumas espécies foram observadas portando epiparasitas na região caudal, como foi o caso de Oikopleura longicauda (Forneris, 1965). Mais recentemente, Vega-Pérez \& Schinke (2008) verificaram a presença do parasita Ellobiopsis sp. na cauda de indivíduos dessa espécie e de $O$. dioica coletados no Sistema estuarino da Baixada Santista, São Paulo.

Os apendiculários são encontrados em todos os oceanos do mundo, sendo a Convergência Subtropical o limite sul de distribuição para muitas espécies. Contudo, são mais abundantes em águas de mistura sobre a plataforma continental e na camada dos primeiros 100 m, além de apresentar maior diversidade de espécies em águas quentes (Bjönberg \& Forneris 1955, Esnal 1978, 1986, 1999).

Os estudos da classe Appendicularia iniciaram-se com Chamisso (1821, In Fenaux, 1993), que descreveu a espécie Appendicularia flagelum Chamisso, 1821 e a incluiu no grupo dos Cnidaria. Posteriormente, foram classificadas como moluscos por Quoy \& Gaimard (1833)" e zoofitos por Quoy \& Gaimard (1833). Até então, esses organismos eram considerados estágios larvais de adultos desconhecidos. Foi Huxley (1851) o primeiro a reconhecer que eram espécimes adultos e os colocou no grupo dos Tunicata. Contudo, para alguns autores continuaram sendo larvas de ascídias ou a geração livre natante de ascídias sésseis. Essas dúvidas foram esclarecidas com o trabalho feito por Fol (1872), baseado na análise do material coletado no Estreito de Messina, Itália, que serviu de base para estudos posteriores que mostraram a grande diversidade de espécies apresentada pela classe (Fenaux 1967).

Gegenbaur (1855) usou o termo Copelata para designar esses organismos, enquanto Balfour (1881, In Fenaux, 1993) Perennichordata, para diferenciá-las dos outros Tunicata. Foi Lahille 
(1888) quem os chamou de Appendicularia e Herdman (1881) de Larvacea. A classificação utilizada no momento foi a proposta por Lohman (1896), embora com pequenas modificações (Fenaux 1967, 1993, 1998).

Os primeiros registros dessas espécies em águas brasileiras foram feitos por Lohmann (1896) a partir de amostras obtidas nas regiões Norte e Nordeste, durante a "Plankton Expedition" realizada em 1889. As coletas de plâncton feitas pela Expedição "Terra Nova" em 1910, nessa última região, permitiram que Garstang \& Georgeson (1935) estudassem a sistemática e distribuição dos apendiculários. Esses mesmos aspectos foram abordados por Lohmann \& Hentschel (1939) baseado no plâncton coletado, ao longo da costa brasileira, nas campanhas oceanográficas feitas pelo N/oc. "Meteor" entre 1925 e 1927 (Forneris 1955, Fenaux 1993, Brandini et al. 1997). Posteriormente, Björnberg \& Forneris $(1955,1958)$ estudaram os apendiculários obtidos durante os cruzeiros do "Baependi" e "Vega", organizados pela Marinha de Guerra Brasileira, à Ilha Trindade (ES). A distribuição do grupo ao redor das Ilhas de Alcatrazes e de Fernando de Noronha foi analisada por Björnberg \& Forneris (1956a,b), enquanto a da região sudeste-sul do Brasil por Forneris $(1957,1965)$.

Os apendiculários da costa Atlântica da América do Sul foram identificados por Fenaux (1968a) a partir de amostras obtidas durante a expedição do "Calypso" realizada em 1961-1962. Anos depois, Esnal \& Castro (1977), Esnal (1978) e Campos (2000) fizeram o levantamento das espécies que ocorrem na região sudeste-sul do Brasil. Mais recentemente, Campos (2004) inventariou as espécies que ocorrem desde Cabo Orange (AM) até o Cabo de Santa Marta Grande-Araranguá (SC) e Bonecker \& Carvalho (2006) identificaram as da região compreendida entre a Bahia e o Rio de Janeiro. Dentre os trabalhos que abordam aspectos da ecologia e abundância desses organismos destacam-se os de Esnal \& Castro (1985), Esnal et al. (1985, 1997) e Dadon \& Esnal (1995). No Estado de São Paulo, Matsumura (1970) estudou a variação sazonal dos apendiculários do Complexo Estuarino-Lagunar de Cananéia e de Santos (SP), Sinque (1976, 1983a, b) a distribuição vertical das famílias Oikopleuridae e Fritillaridae ao largo de Santos, enquanto Vega-Pérez \& Schinke (2008) e Miyashita (2010) as que ocorrem no sistema estuarino de Santos e na região de Ubatuba, respectivamente.

Das 82 espécies identificadas até o momento em todo o mundo, 43 foram citadas para o Oceano Atlântico Sul e 36 para a costa brasileira por Esnal (1999). Baseado nos mapas de distribuição dos apendiculários, elaborados por essa autora, Rodrigues (1999) concluiu que 25 espécies encontram-se presentes na faixa latitudinal correspondente ao Estado de São Paulo. Entretanto, a análise dos trabalhos feitos por pesquisadores brasileiros, com material coletado em várias regiões do Brasil e em épocas diferentes, revelam a existência de 29 espécies, sendo o maior número registrado na região compreendida entre Cabo Frio (RJ) e Cabo de Santa Marta Grande (SC) (Campos 2000, 2004).

\section{Metodologia}

A lista das espécies da fauna de Appendicularia do Estado de São Paulo (Tabela 1) foi elaborada com base nos trabalhos dos seguintes autores: Forneris (1957); Björnberg (1963); Björnberg \& Forneris (1955, 1956a,b, 1958); Matsumura-Tundisi (1970), Esnal (1981, 1999); Campos (2000; 2004), Campos \& Vega-Pérez (2004), VegaPérez \& Schinke (2008) e Miyashita (2010). Além dessas referências foram consultados os seguintes sites:

- World Register of Marine Species - WORMS: www.marinespecies.org/. Acessado em 10.05.2010.

- Ocean Biogeographic Information System - OBIS: www.iobis. org/. Acessado em 10.05.2010.

\section{Resultados e Discussão}

\section{Lista das espécies da classe appendicularia \\ (Chordata: Tunicata) do Estado de São Paulo}

\author{
Filo Chordata \\ Subfilo Tunicata \\ Classe Larvacea ou Appendicularia \\ Família Oikopleuridae Lohmann, 1915 \\ Oikopleura albicans (Leuckart, 1853) \\ Oikopleura cophocerca (Gegenbaur, 1855) \\ Oikopleura dioica Fol 1872 \\ Oikopleura fusiformis cornutogastra Aida 1907 \\ Oikopleura fusiforms Fol 1872 \\ Oikopleura gracilis Lohmann 1896 \\ Oikopleura intermedia Lohmann 1896 \\ Oikopleura longicauda (Vogt, 1854) \\ Oikopleura rufescen Fol 1872 \\ Stegosoma magnum (Langerhans, 1880) \\ Família Fritillariidae Lohmann, 1915 \\ Appendicularia sicula Fol 1874 \\ Fritillaria borealis sargassi Lohmann 1896 \\ Fritillaria borealis Lohmann 1896 \\ Fritillaria formica Fol 1872 \\ Fritillaria haplostoma Fol 1872 \\ Fritillaria pellucida (Busch 1851) \\ Fritillaria tenella Lohmann 1896 \\ Fritillaria venusta Lohmann 1896 \\ Tectillaria fertilis (Lohmann, 1896) \\ Família Kowalevskiidae Lahille, 1888 \\ Kowalevskia tenuis Fol,1872
}

\section{Comentários sobre a lista, riqueza do estado comparado com outras regiões}

A fauna de Appendicularia registrada no ecossistema marinho do Estado de São Paulo é muito semelhante à encontrada no mundo e em outras regiões do Brasil, como as compreendidas entre Bahia e Rio de Janeiro, bem como entre o Rio de Janeiro e Santa Catarina, onde Bonecker \& Carvalho (2006) e Campos (2004) reportaram a presença de 15 e 17 espécies, respectivamente. O fato de São Paulo ter apresentado $20(69 \%)$ das 29 espécies registradas em águas brasileiras permite inferir que, no momento, é o estado que possui a fauna mais diversificada e melhor conhecida do país.

\section{Principais avanços relacionados ao Programa BIOTA/FAPESP}

Apesar da importância dos apendiculários no ecossistema marinho, nos últimos 10 anos pouco foi feito para melhorar o conhecimento do grupo no Estado de São Paulo. A prova desse fato é o pequeno número de trabalhos publicados em periódicos nacionais e internacionais, de teses (01) e dissertações apresentadas (02). Estas últimas foram elaboradas por alunos do curso de Pós-graduação do IOUSP e defendidas nos anos 2000, 2010 e 2004, respectivamente. Verifica-se também que entre 1999 e 2010 nenhuma monografia de conclusão de curso foi apresentada.

\section{Principais grupos de pesquisa}

No Brasil e no Estado de São Paulo é muito reduzido o número de pesquisadores dedicados ao estudo dos apendiculários. No momento dois grupos pertencentes aos Laboratórios de Zooplâncton e de Sistemas planctônicos, do Instituto Oceanográfico da Universidade de São Paulo, vêm pesquisando a fauna de Appendicularia. 
Vega-Pérez, L.A. et al.

Tabela 1. Espécies de Appendicularia encontradas nas águas brasileiras.

Table 1. Species of Appendicularia found in Brazilian waters.

\begin{tabular}{|c|c|c|c|c|c|}
\hline Subfilo & Classe & Gênero & Epíteto específico & Descritor & Ano da descrição \\
\hline \multirow[t]{29}{*}{ Tunicata } & Appendicularia & Folia & gracilis & Lohmann & 1892 \\
\hline & & Megalocercus & abyssorum & Chun & 1887 \\
\hline & & Oikopleura & albicans $*$ & (Leuckart) & -1853 \\
\hline & & Oikopleura & cophocerca* & (Gegenbaur) & -1855 \\
\hline & & Oikopleura & dioica * & Fol & 1872 \\
\hline & & Oikopleura & fusiformis $*$ & Fol & 1872 \\
\hline & & Oikopleura & fusiformis $\mathrm{f}$. cornutogastra * & Aida & 1907 \\
\hline & & Oikopleura & gracilis $*$ & Lohmann & 1896 \\
\hline & & Oikopleura & intermedia * & Lohmann & 1896 \\
\hline & & Oikopleura & longicauda * & (Vogt) & -1854 \\
\hline & & Oikopleura & parva & Lohmann & 1896 \\
\hline & & Oikopleura & rufescens $*$ & Fol & 1872 \\
\hline & & Stegosoma & magnum * & (Langerhans) & -1880 \\
\hline & & Appendicularia & sicula* & Fol & 1874 \\
\hline & & Fritillaria & aequatorialis & Lohmann & 1896 \\
\hline & & Fritillaria & antarctica & Lohmann & 1905 \\
\hline & & Fritillaria & borealis $*$ & Lohmann & 1896 \\
\hline & & Fritillaria & borealis f. sargassi ${ }^{*}$ & Lohmann & 1896 \\
\hline & & Fritillaria & formica $*$ & Fol & 1872 \\
\hline & & Fritillaria & fraudax & Lohmann & 1896 \\
\hline & & Fritillaria & gracilis & Lohmann & 1896 \\
\hline & & Fritillaria & haplostoma $*$ & Fol & 1872 \\
\hline & & Fritillaria & megachile & Fol & 1872 \\
\hline & & Fritillaria & messanensis & Lohmann in Bückmann & 1924 \\
\hline & & Fritillaria & pellucida $*$ & (Busch) & -1851 \\
\hline & & Fritillaria & tenella* & Lohmann & 1896 \\
\hline & & Fritillaria & venusta $*$ & Lohmann & 1896 \\
\hline & & Tectillaria & fertilis * & (Lohmann) & -1896 \\
\hline & & Kowalevskia & tenuis $*$ & Fol & 1872 \\
\hline
\end{tabular}

(*) ocorrem no estado de São Paulo.

\section{Principais acervos}

Até o momento, não existem coleções de referência completas da fauna de Appendicularia que ocorrem em águas brasileiras. Os Laboratórios de Zooplâncton e o de Sistemas planctônicos do Instituto Oceanográfico da Universidade de São Paulo possuem uma coleção de espécimes retirados das amostras de zooplâncton, pertencentes ao acervo da instituição, além de uma pequena coleção didática.

\section{Principais lacunas do conhecimento}

Apesar do número de espécies de Appendicularia do estado de São Paulo representar $69 \%$ das 29 encontradas em águas brasileiras, $46 \%$ das 43 registradas no Atlântico Sul e $24 \%$ das 82 espécies descritas no mundo, a sistemática do grupo ainda precisa ser aperfeiçoada. Isso, aliado ao estudo detalhado da distribuição batimétrica desses organismos, pode revelar a ocorrência de maior número de espécies no estado.

Os apendiculários foram estudados de um modo geral a partir de amostras coletadas esporadicamente ao longo da costa brasileira em profundidades de até $200 \mathrm{~m}$, existindo poucos registros disponíveis para as regiões de Ubatuba, São Sebastião e os sistemas estuarinos de Santos e Cananéia. Assim é fundamental que sejam intensificados os estudos nesses locais, em regiões oceânicas, em várias profundidades e também em locais fixos, o que pode aumentar o registro do número de espécies no Estado de São Paulo.

Com relação à metodologia, a coleta desses organismos exige a utilização de equipamento específico, os quais superam a performance das redes na captura desses organismos que são muito pequenos, delicados e frágeis.

É necessária a formação de uma coleção de referência dos apendiculários para facilitar os trabalhos de sistemática feitos por pesquisadores e pelos alunos de graduação e de pós-graduação interessados em estudar esses organismos marinhos.

O estudo detalhado do grupo permitiria, também, conhecer o número real de espécies que ocorrem no Estado de São Paulo e fazer monitoramento faunístico que permita detectar, com o tempo, espécies em extinção, invasoras ou introduzidas, e a existência de endemismo.

\section{Perspectivas de pesquisa em classe Appendicularia (Chordata: Tunicata) para os próximos 10 anos}

O número de espécies que ocorrem em águas brasileiras está longe de ser totalmente conhecido devido, principalmente, ao reduzido número de pesquisadores dedicados ao estudo do grupo. Portanto, é necessário incentivar a formação de especialistas, mediante a 
concessão de bolsas de estudo e de políticas que favoreçam sua permanência no Estado de São Paulo, a fim de que possam dar continuidade aos estudos dos apendiculários.

Esforços devem ser feitos para a criação, manutenção e informatização de coleções didáticas e de referência, o que facilitaria o trabalho dos pesquisadores dedicados à taxonomia do grupo e ajudaria a despertar o interesse de alunos de graduação e de pós-graduação pelo estudo dos apendiculários. Além disso, é importante estimular o desenvolvimento de estudos taxonômicos com material já coletado e que, ainda, não foi analisado.

Ênfase deve ser dada ao estudo da fauna que habita as regiões oceânicas, em várias profundidades, e estuarinas a fim de poder inventariar as espécies que ocorrem em São Paulo, porque é muito provável que outras espécies venham a ser encontradas. Para atingir essas metas é fundamental que projetos multidisciplinares, ou mesmo individuais, que tenham como objetivo o estudo de invertebrados marinhos e que apresentem planejamento minucioso recebam apoio financeiro. Atenção especial deve ser dada à infra-estrutura necessária às coletas desses organismos, as quais exigem utilização de equipamento específico, bem como a participação de técnicos especializados para realização dos trabalhos de campo e laboratório. Apoio financeiro também deve ser dado para a modernização de laboratórios, equipamentos para análise das amostras e para publicação dos resultados em revistas internacionais e nacionais.

\section{Referências Bibliográficas}

A bibliografia apresentada a seguir, foi utilizada na elaboração do texto apresentado acerca da Classe Appendicularia. Grande parte da bibliografia pode ser encontrada nas bibliotecas do Instituto Oceanográfico, Instituto de Biociências, Centro de Biologia Marinha e Museu de Zoologia da Universidade de São Paulo.

ALLDREDGE, A.L. \& MADIN, L.P. 1982. Pelagic Tunicates: unique herbivorous in the marine plankton. Bioscience 32:655-663.

ALLDREDGE, A.L. 1976a. Field behavior and adaptative strategies of Appendicularians (Chordata: Tunicata). Mar. Biol. 38:29-39. http://dx.doi. org/10.1007/BF00391483

ALLDREDGE, A.L. 1976b. Appendicularians. Scient. Am. 235:95-102. http:// dx.doi.org/10.1038/scientificamerican0776-94

ALLDREDGE, A.L. 1977. House morphology and mechanisms of feeding in the Oikopleuridae (Tunicata, Appendicularia). J. Zool. Proc. Soc. Lond., 181:175-188.

ALLDREDGE, A.L. 2005. The contribution of descarted appendicularian house to their flux of particulate organic carbon from oceanic surface waters. In Response of marine ecosystems to global change: Ecological impact of appendicularians (G. Gorsky \& D. Deibel, eds.). Gordon and Breach Scientific Publishers, Paris, p.309-326.

BARHAM, E.G. 1979. Giant larvacean houses: observations from deep submersibles. Science, 2005:1129-1131.

BARNES, R.S. \& HUGHES, R.N. 1982. An introduction to marine ecology. Blackwell, Oxford.

BERRIL, N.J. 1950. The Tunicata with an account of the British species. London: Adlard \& Son, p.302-322.

BJÖRNBERG, T.K.S. \& FORNERIS, L. 1955. Resultados científicos do Cruzeiro do "Baependî" e do "Vega" à Ilha de Trindade - Copelata I Contrções Inst. oceanogr., Univ. S. Paulo, 1:68p.

BJÖRNBERG, T.K.S. \& FORNERIS, L. 1956a. On the uneven distribution of the Copelata of the Alcatrazes area. Bolm. Inst. Oceanogr. 7(1-2):113-115. http://dx.doi.org/10.1590/S0373-55241956000100007

BJÖRNBERG, T.K.S. \& FORNERIS, L. 1956b. On the uneven distribution of the Copelata of the Fernando de Noronha area. Bolm. Inst. Oceanogr. 7(1-2):105-111. http://dx.doi.org/10.1590/S0373-55241956000100006
BJÖRNBERG, T.K.S. \& FORNERIS, L. 1958. Resultados cientificos de los cruceros del "Baependî" y del "Vega" a la Isla Trindade - Copelata II. Neotropica 4(15):81-85.

BJÖRNBERG, T.K.S. 1963. On the marine free living copepods off Brazil. Bolm. Inst. Oceanogr., S Paulo 13(1):3-142.

BONE, Q., CARRÉ, C. \& CHANG, P. 2003. Tunicate feeding filters. J. Mar. Biol. Ass. U.K., 83:907-919.

BONECKER, S.L.C. \& CARVALHO, P.F. 2006. Appendicularia. In Atlas de zooplâncton da região central da zona econômica exclusiva brasileira (S.L.C. Bonecker, ed.). Museu Nacional, Rio de Janeiro, p.185-202.

BRANDINI, F.P., LOPES, R.M., GUTSEIT, K.S., SPACH, H. L. \& SASSI, R. 1997. Planctologia na plataforma continental do Brasil - Diagnose e revisão bibliográfica. Avaliação do Potencial Sustentável de Recursos Vivos na Zona Econômica Exclusiva- REVIZEE. 196p.

BRUSCA, R.C. \& BRUSCA, G.J. 2007. Invertebrados. 2. ${ }^{\text {a }}$ ed. Editora Guanabara Koogan, Rio de Janeiro.

CAMPOS, M.A.G. \& VEGA-PÉREZ, L.A. 2004. First records of Fritillaria venusta Lohmann, 1896 (Tunicata: Appendicularia) in South Brazil Bight. Oceánides 19(1):11-17.

CAMPOS, M.A.G. 2000. As Appendicularia (Chordata: Tunicata) da região compreendida entre Cabo Frio (RJ) e Cabo de Santa Marta Grande (SC). Dissertação de mestrado, Universidade de São Paulo, São Paulo.

CAMPOS, M.A.G. 2004. Apendiculárias da costa brasileira. Tese de doutorado, Universidade de São Paulo, São Paulo.

CAPITANIO, F., PÁJARO, M. \& ESNAL, G.B. 1997. Appendicularians (Chordata, Tunicata) in the diet of anchovy (Engraulis anchoita) in the Argentine Sea. Sci. Mar. 61(1):9-15.

CHOE, N. \& DEIBEL, D. 2008. Temporal and vertical distributions of three appendicularian species (Tunicata) in Conception Bay, Newfoundland. J. Plankt. Res. 30(9):969-979. http://dx.doi.org/10.1093/plankt/fbn064

CIMA, F., BRENA, C. \& BURIGHEL, P. 2002. Multifarious activities of gut epithelium in an appendicularian (Oikopleura dioica: Tunicata). Mar. Biol. 141:479-490. http://dx.doi.org/10.1007/s00227-002-0850-5

DADON, J.R. \& ESNAL, G.B. 1995. Abundance and assemblages of planktonic Tunicata (Chordata) and Thecosomata (Mollusca) off South Brazil. Physis 50(118-119):25-30.

DAGG, M.J. \& BROWN, S.L. 2005. The potential contribution of fecal pellets from larvacean Oikopleura dioica to vertical flux of carbon in a river dominated coastal margin. In response of marine ecosystems to global change: ecological impact of appendicularians. (G. Gorsky, M.J. Youngbluth \& D. Deibel, eds). Contemporary Publishing International, Paris, p.293-308.

DAGG, M.J. GREEN, E.P., McKEE, B.A. \& ORTNER, P.B. 1996. Biological removal of fine-grained lithogenic particles from a large river plume. J. mar. Res., 54:149-160.

DAVOLL, P.J. \& YOUNGBLUTH, M.J. 1990. Heterotrophic activity on appendicularian (Tunicata: Appendicularia) houses in mesopelagic regions and their potential contribution to particle flux. Deep-Sea Res. 37(2):285-294. http://dx.doi.org/10.1016/0198-0149(90)90128-I

DEIBEL, D. \& PAFFENHÖFER, G.-A. 2009. Predictability of patches of neritic salps and doliolids (Tunicata, Thaliacea). J. Plank. Res., 31(12):1571-1579. http://dx.doi.org/10.1093/plankt/fbp091

DEIBEL, D. 1988. Filter feeding by Oikopleura vanhoeffeni: grazing impact on suspended particles in cold ocean waters. Mar. Biol. 99:177-186.

DYBAS, C.L. 1993. Beautiful, ethereal Larvaceans play a central role in Ocean Ecology. Oceanus 84-86.

ESNAL, G.B 1999. Appendicularia. In South Atlantic zooplankton (D. Boltovskoy, ed.) Backhuys Publishers, Leiden, The Netherlands, v.2, p.1375-1399.

ESNAL, G.B. \& CASTRO, R.J. 1977. Distributional and biometrical study of Appendicularians from the West South Atlantic Ocean. Hydrobiologia 56(3):241-246. http://dx.doi.org/10.1007/BF00017510

ESNAL, G.B. \& CASTRO, R.J. 1985. Caracterizacion de estadios de madurez y variaciones intraespecificas in Oikopleura longicauda (Vogt, 1854) (Tunicata, Appendicularia). Physis, Secc. A 43(104):19-24. 
ESNAL, G.B. 1981. Appendicularia. In Atlas del zooplancton del Atlántico Sudoccidental y métodos de trabajo con zooplancton marino (D. Boltovskoy, ed.). Mar del Plata, Inst. Nacional Invest. Desarrollo Pesquero, INIDEP, p.809-827.

ESNAL, G.B. 1986. Tunicados planctonicos (Chordata, Tunicata), distribucion y relaciones troficas. Physis, Secc. A, 44(106):51-57.

ESNAL, G.B. 1996. Thaliacea e Appendicularia. In Introducción al estudio del zooplancton marino (R. Gasca \& E. Suárez, ed.). México: El Colegio de la Frontera Sur (ECOSUR)/CONACYT, 711p.

ESNAL, G.B., SANKARANKUTTY, C. \& CASTRO, R.J. 1985. Diurnal and seasonal fluctuations of Oikopleura dioica Fol, 1872 (Tunicata, Appendicularia) in the mouth of the River Potengi (North Brazil). Physis, Secc. A 43(105):65-71.

ESNAL, G.B., SANKARANKUTTY, C., GONZALEZ, B.N. \& CAPITANIO, F.L. 1997. Comparative study on the efficiency of two plankton nets for the quantitative estimation of appendicularians (Chordata, Tunicata). Neritica 11:9-16.

FENAUX, R. 1967. Les appendiculaires des mers d'Europe et du basin méditerranéen. Masson et Cie., 2:1-116.

FENAUX, R. 1968a. Appendiculaires. Annls Inst. océanogr., Paris 2:33-46.

FENAUX, R. 1977. Life history of the appendicularians (genus Oikopleura). Proceedings of the symposium on warm zooplankton. National Institute of Oceanography, Goa, p.497-510.

FENAUX, R. 1993. The classification of Appendicularia (Tunicata): history and current state. Mém. Inst. océanogr., Monaco, 17, 123p.

FENAUX, R. 1993. The classification of Appendicularia (Tunicata): history and current state. Mém. Insttit. Oceanogr. Mónaco, 17(7):127p.

FENAUX, R., BONE, Q. \& DEIBEL, D. 1998. Appendicularian distribution and zoogeography. In The biology of pelagic tunicates (Q. Bone, ed.). Oxford University Press, Oxford, p.295-306.

FERNÁNDEZ, D. \& ACUÑA, J.L. 2003. Enhancement of marine phytoplankton blooms by appendicularian grazers. Limnol. Oceanogr. 48(2):587-593. http://dx.doi.org/10.4319/lo.2003.48.2.0587

FLOOD, P.R. 1978. Filter characteristics of appendicularians food catching nets. Cel. Mol. Life Sci., 34(2):173-175.

FLOOD, P.R. 1991. Architecture of, and water circulation and flow rate in, the house of the planktonic tunicate Oikopleura labradoriensis. Mar. Biol. 111:95-111.

FLOOD, P.R. 2003. House formation and feeding behaviour of Fritillaria borealis (Appendicularia: Tunicata). Mar. Biol., 143:467-475.

FLOOD, P.R., DEIBEL, D. \& MORRIS, C.C. 1992. Filtration of colloidal melanin from sea water by planktonic tunicates. Nature 355:630-632. http://dx.doi.org/10.1007/BF01986351

FOL, H. 1872. Études sur les appendiculaires du détroit de Messine. Mémoires de la Societé de Physique et d'Histoire Naturelle de Gèneve, 21:445-499.

FORNERIS, L. 1957. The geographical distribution of the Copelata. An. Acad. Bras. Ciênc. 29:273-284.

FORNERIS, L. 1965. Appendicularian species groups and Southern Brazil water masses. Bolm. Inst. Oceanogr. S Paulo 14:53-114.

GARSTANG, W. \& GEORGESON, E. 1935. Report on the Tunicata. Part II. Copelata. Natural History Report - British Antarctic ("Terra Nova") Expedition, 1910 (Zoology), 4(8):268-282.

GEGENBAUR, C. 1855. Bemerkungen über die organisation der Appendicularien. Zeitschrift für Wissenschaftliche Zoologie, 6(3/4):406427.

GORKY, G. \& FENAUX, R. 2003. The role of Appendicularia in marine food webs. In The biology of pelagic tunicates. (Q. Bone, ed). Oxford University Press, Oxford, p.161-170.

GORKY, G. \& PALAZZOLI, I. 1989. Aspects de la biologie de l'Appendiculaire Oikopleura dioica Fol 1872 (Chordata: Tunicata). (Dynamique du plancton gélatineux. Nice-Acrópolis, 27-28 Octobre 1988). Océanis, 15(1):39-49.
HERDMAN, W.A. 1988. Report upon the Tunicata collected during the voyage of H.M.S. Challenger during the years 1873-76. Part 3. Report on the scientific results of the voyage of H.M.S. Challenger during the years 1873-76, zoology, 27(4):1-166.

HOPCROFT, R.R. \& ROBISON, B.H. 2005. New mesopelagic larvaceans in the genus Fritillaria from Monterey Bay, California. J. Mar. Biol. Ass. U.K. 85:665-678. http://dx.doi.org/10.1017/S0025315405011598

HOPCROFT, R.R. \& ROFF, J.C. 1995. Zooplankton growth rates: extraordinary production by the larvacean Oikopleura dioica in tropical waters. J. Plankt. Res. 17(2):205-220. http://dx.doi.org/10.1093/ plankt/17.2.205

HOPCROFT, R.R. \& ROFF, J.C. 1998. Production of tropical larvaceans in Kingston Harbour, Jamaica: are we ignoring an important secondary producer? J. Plankt. Res. 20(3):556-569. http://dx.doi.org/10.1093/ plankt/20.3.557

HOPCROFT, R.R., ROFF, J.C. \& BOWMAN, H.A. 1998. Zooplankton growth rates: the larvaceans Appendicularia, Fritillaria and Oikopleura in Tropical waters. J. Plankt. Res. 20(3):539-555. http://dx.doi.org/10.1093/ plankt/20.3.539

HUXLEY, T.H. 1851. Observations upon the anatomy and physiology of salpa and Pyrosoma. Philosophical transactions of the Royal Society of London, 14(2):567-594

IKEWAKI, Y. \& TANAKA, M. 1993. Feeding habitats of Japanese flounder (Paralichthys olivaceus) larvae in the Western part of Wakasa Bay, the Japan Sea. Nippon Suisan Gakkaishi 59(6):951-956. http://dx.doi. org/10.2331/suisan.59.951

KING, K.R. 1982. The population biology of the larvacean Oikopleura dioica in enclosed water columns. In Marine mesocosms: Biological and chemical research in experimental ecosistems (G.D. Grice, ed.). Spring-Verlag, Berlin, p.341-351.

KING, K.R., HOLLIBAUGH, J.T. \& AZAM, F. 1980. Predator-prey interactions between the larvacean Oikopleura dioica and bacterioplankton in enclosed water columns. Mar. Biol, 56:49-57.

KOSKI, M., MØLLER, E.F., MAAR, M. \& VISSER, A.W. 2007. The fate of discarded appendicularian houses: degradation by the copepod, Microsetella norvegica, and other agents. J. Plankt. Res. 29(7):641-654. http://dx.doi.org/10.1093/plankt/fbm046

KURTZ, F.W. 1999. Dinâmica larval de Sardinella brasiliensis (Steindachner, 1879) (Teleostei, Clupeidae) na Região Sudeste do Brasil e implicações no recrutamento. Tese de doutorado, Universidade de São Paulo, São Paulo.

LAHILLE, F. 1888. Étude systématique des Tuniciers. Compte rendu de la 16e session. Toulouse, 1887. Association française pour l'avancement des sciences, 2:667-677.

LAST, J.M. 1972. Egg development, fecundity and growth of Oikopleura dioica Fol in the North Sea. J. Cons. int. Explor. Mer 34(2):232-237.

LAST, J.M. 1980. The food of twenty species of fish larvae in the west central North Sea. Fish Res. Tech. Rept., 60: 44p.

LIANG, T.H. \& VEGA-PÉREZ, L.A. 1995. Studies on chaetognaths of Ubatuba region, Brazil. II. Feeding habits. Bolm Inst. oceanogr., S Paulo, 43(1):27-40.

LIANG, T.H. \& VEGA-PÉREZ, L.A. 2001. Diversity, abundance, and biomass of epiplanktonic chaetognath off South Atlanctic Western sector, from Cabo Frio (230 S, 420 W) to São Pedro and São Paulo Rocks (01o N, 29o W). Oceanides, 16(1):34-48.

LIANG, T.H. \& VEGA-PÉREZ, L.A. 2002. Distribution, abundance and biomass of chaetognaths off São Sebastião region, Brazil, in February 1994. Rev. Bras. Oceanogr., 50(único):1-12.

LOHMAN, H. \& HENTSCHEL, E. 1939. Die Appendicularien der Südatlantischen Ozean. Wissenschaftliche Ergebnisse der Deutschen Atlantischen Expedition auf dem Forschungs-und Vermessungsschiff "Meteor" 1925-1927, 13(3):153-243.

LOHMAN, H. 1896. Die Appendicularien der Plankton-Expedition. Ergebnisse der Plankton-Expedition der Humboldt-Stiftung, 2:1-148.

LOHMANN, H. 1896. Die appendicularien der plankton-expedition. Ergebnisse der Plankton-Expedition der Humboldt Siboga 2:1-148. 
LOPES-URRUTIA, Á., HARRIS, R.P., ACUÑA, J.L., BAMSTEDT, U., FLOOD, P.R., FYHN, H.J., GRASSER, B., GORSKY, G., IRIGOIEN, X. \& MARTINUSSEN, M.B. 2005. A comparison of appendicularian seasonal cycles in four distinct European coastal environments.In Response of marine Ecosystems to Gloval Change: Ecological Impact of Appendicularians. (G. Gorsky, M.J.Youngbluth, D. Deibel, eds). Contemporary Publishing International, Paris, France, p.255-276.

LOPES-URRUTIA, Á., IRIGOIEN, X., ACUÑA, J.L.,HARRIS, R. 2003. Food limitation and growth in temperate epipelagic appendicularians (Tunicata). Mar. Ecol.-Prog.Ser., 252:143-160.

LÓPEZ-URRUTIA, Á., IRIGOIEN, X., ACUÑA, J.L. \& HARRIS, R. 2003a. Food limitation and growth in temperate epipelagic appendicularians (Tunicata). Mar. Ecol. -Prog. Ser. 252:143-157. http://dx.doi.org/10.3354/ meps 252143

LÓPEZ-URRUTIA, Á., IRIGOIEN, X., ACUÑA, J.L. \& HARRIS, R. 2003b. In situ feeding physiology and grazing impact of the appendicularian community in temperate waters. Mar. Ecol. -Prog. Ser. 252:125-141. http://dx.doi.org/10.3354/meps252125

MAAR, M., NIELSEN, T.G., GOODING, S., TÖNNESSON, K., TISELIUS, P., ZERVOUDAKI, S., CHRISTOU, E., SELL, A. \& RICHARDSON, K. 2004. Trophodynamic function of copepods, appendicularians and protozooplankton in the late summer zooplankton community in the Skagerrak. Mar. Biol. 144:917-933. http://dx.doi.org/10.1007/s00227003-1263-9

MATSUMURA-TUNDISI, T. 1970. On the seasonal occurrence of appendicularians in waters off the coast of São Paulo state. Bolm.Inst. Oceanogr. 19:131-144

MIYASHITA, L.K. 2010. Dinâmica populacional de Appendicularia e Cladocera na plataforma interna de Ubatuba: um estudo sazonal e multianual. Dissertação de mestrado, Universidade de São Paulo, São Paulo.

OCEAN BIOGEOGRAPHIC INFORMATION SYSTEM - OBIS: www.iobis org/ (último acesso em 25/05/2010.

PURCELL, J.E., STUDERVANT, M.V. \& GALT, C.P. 2005. A review of appendicularians as prey of invertebrate and fish predators. In Responses of marine ecosystem to global change: ecological impact of appendicularians (G. Gorsky, M.J. Youngbluth. \& D. Deibel, ed.). Contemporary Publishing International, Paris, p.359-434.

RODRIGUES, S.A. 1999. Appendicularia ou Larvacea. In Biodiversidade do Estado de São Paulo, Brasil: Síntese do conhecimento ao final do século XX. Invertebrados Marinhos. (C.A. Joly \& C.E.M. Bicudo, ed.). FAPESP, São Paulo, v.3, p.277-284.

SATO, R., ISHIBASHI, Y., TANAKA, Y., ISHIMARU, T. \& DAGG, M.J. 2008. Productivity and grazing impact of Oikopleura dioica (Tunicata, Appendicularia) in Tokyo bay. J. Plankt. Res. 30(3):299-309. http://dx.doi. org/10.1093/plankt/fbn001

SATO, R., TANAKA, Y. \& ISHIMARU, T. 2001. House production by Oikopleura dioica (Tunicata, Appendicularia) under laboratory conditions. J. Plankt. Res. 23(4):415-423. http://dx.doi.org/10.1093/ plankt/23.4.415
SATO, R., TANAKA, Y. \& ISHIMARU, T. 2003. Species-specific house productivity of appendicularians. Mar. Ecol. Prog. Ser. 259:163-172. http://dx.doi.org/10.3354/meps259163

SINQUE, C. 1976. A migração vertical das Appendicularia (Tunicata) ao largo de Santos (Brasil). Dissertação de mestrado, Universidade de São Paulo, São Paulo.

SINQUE, C. 1983a. Distribuição vertical de Appendicularia (Tunicata) ao largo de Santos, Brasil (24 16’08'S e 46 00'04”'W). Oikopleura. Arq. Biol. Tecnol. 26(1):61-76.

SINQUE, C. 1983b. Distribuição vertical de Appendicularia (Tunicata) ao

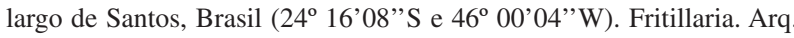
Biol. Tecnol. 26(3):359-371.

TAGUCHI, S. 1982. Seasonal study of fecal pellets and discarded houses of Appendicularia in a subtropical inlet, Kaneohe Bay, Hawaii. Estuar. Coast. Shelf Sci. 14:545-555.

TOMITA, M., IKEDA, T. \& SHIGA, N. 1999. Production of Oikopleura longicauda (Tunicata: Appendicularia) in Toyama Bay, Southern Japan Sea. J. Plankt. Res. 21(12):2421-2430. http://dx.doi.org/10.1093/ plankt/21.12.2421

URBAN, J.L., DEIBEL, D. \& SCHWINGHAMER, P. 1993. Seasonal variations in the densities of fecal pellets produced by Oikopleura vanhoeffeni (C. Larvacea) and Calanus finmarchicus (C. Copepoda). Mar. Biol. 117:607-613. http://dx.doi.org/10.1007/BF00349772

URBAN, J.L., MAKENZIE, C.H. \& DEIBEL, D. 1992. Seasonal differences in the content of Oikopleura vanhoeffeni and Calanus finmarchicus faecal pellets: ilustrations of zooplankton food web shifts in coastal Newfoundland waters. Mar. Ecol. -Prog. Ser. 84:255-264. http://dx.doi. org/10.3354/meps 317101

URBAN-RICH, J., FERNÁNDEZ, D., ACUÑA, J.L. 2006. Grazing impact on chromophoric dissolved organic matter $(\mathrm{CDOM})$ by the larvacean Oikopleura dioica. Mar. Ecol.Prog.Ser. 317:101-110.

UYE, S. \& ICHINO, S. 1995. Seasonal variations in abundance, size composition, biomass and production rate of Oikopleura dioica (Fol) (Tunicata: Appendicularia) in a temperate eutrophic inlet. J. Exp. Mar. Biol. Ecol. 189:1-11. http://dx.doi.org/10.1016/0022-0981(95)00004-B

VARGAS, C.A., TÖNNESSON, K., SELL, A., MAAR, M., MØLLER, E.F., ZERVOUDAKI, T., GIANNAKOUROU, A., CHRISTOU, E., SATAPOOMIN, S., PETERSEN, J.K., NIELSEN, T.G. \& TISELIUS, P. 2002. Importance of copepods versus appendicularians in vertical carbon fluxes in a Swedish fjord. Mar. Ecol. -Prog. Ser. 241:125-138. http:// dx.doi.org/10.3354/meps241125

VEGA-PÉREZ, L.A. \& SCHINKE, K.P. 2008. Zooplâncton. In Influência do complexo estuarino da Baixada santista sobre o ecossistema da Plataforma adjacente - ECOSAN. (A.M.S. Pires-Vanin, coord.). Rel. $\mathrm{n}^{\circ} 4$ (julho 2007- junho 2008), cap.5, p.270-306.

WEFER, G., FISCHER, G., FÜETTERER, D. \& GERSONDE, R. 1988. Seasonal particle flux in the Bransfield Strait, Antarctica. Deep-Sea Res. 35:891-898. http://dx.doi.org/10.1016/0198-0149(88)90066-0

WORLD REGISTER OF MARINE SPECIES - WORMS: wWw. marinespecies.org/ (último acesso em 25/05/2010.

Recebido em 23/07/2010

Versão reformulada recebida em 08/10/2010

Publicado em 15/12/2010 
Vega-Pérez, L.A. et al.

\section{Apêndice}

Apêndice 1. Referências complementares.

Appendix 1. Complementary references.

ACUÑA, J.L. 1994. Summer vertical distribution of Appendicularians in the Central Cantabrian Sea (Bay of Biscay). J. Mar. Biol. Ass. U.K. 74:585-601. http://dx.doi.org/10.1017/S0025315400047688

ACUÑA, J.L. 2001: Pelagic tunicates:why gelatinous? Am. Nat. 158:100-107. http://dx.doi.org/10.1086/320864

ACUÑA, J. L. \& ANADÓN, R. 1992. Appendicularian assemblages in a shelf area and their relationship with temperature. J. Plankt. Res. 14(9):1233-1250. http://dx.doi.org/10.1093/plankt/14.9.1233

ACUÑA, J.L., BESO, A.W., HARRIS, R.P. \& ANADÓN, R. 1995. The seasonal succession of appendicularians (Tunicata: Appendicularia) off Playmouth. Mar. Biol. Ass. U.K. 75:755-758. http://dx.doi.org/10.1017/S0025315400039187

AGUIRRE, G.E., CAPITANIO, F.L., VIÑAS, M.D \& ESNAL, B.G. 2006. Gonadal development, allometric growth and ecological impact of Appendicularia sicula (Appendicularia: Fritillaridae) from the south-western Atlantic Ocean. J. Mar. Biol. Ass. UK. 86:1215-1220. http://dx.doi.org/10.1017/S0025315406014214

ALLDREDGE, A.L. 1982. Aggregation of spawning appendicularians in surface windrows. Bull. Mar. Sci. 32(1):250-254.

ALLDREDGE, A.L. 1984. The quantitative sgnificance of gelatinous zooplankton as pelagic consumers. In Flows of energy and material in marine ecosystem. Theory and practice (M.J.R. Fasham, ed.). Plenum Press, New York, p.407-433. http://dx.doi.org/10.1007/978-1-4757-0387-0_16

ALLDREDGE, A.L. 2000. Interstitial dissolved organic carbon (DOC) concentrations within sinking marine aggregates and their potential contribution to carbon flux. Limnol. Oceanogr. 45:1245-1253. http://dx.doi.org/10.4319/lo.2000.45.6.1245

BARY, B.M. 1960. Notes on ecology, distribution, and systematics of pelagic Tunicata from New Zealand. Pacif. Sci. 14:101-121.

BINET, D. 1976. Contribution à Y’écologie de quelques taxons du zooplancton de Côte D`Ivoire. II. Dolioles, Salpes, Appendiculaires. Doc. Scient. Cent. Rech. Océanogr, Abidjan 7(1):45-61.

BOLTOVSKOY, D. 1981. Caracteristicas biologicas del Atlantico Sudoccidental. In Atlas de zooplancton del Atlantico Sudoccidental y metodos de trabajo com zooplancton marino (D. Boltovskoy, ed.). Inst. Nacional Invest. Desarrollo Pesquero, Mar del Plata, p.239-251.

BONE, Q. 2003. The biology of pelagic tunicates. Oxford University Press, Oxford.

BONECKER, S.L.C., NOGUEIRA, C.R., BONECKER, A.C.T., SANTOS, L.H.S., REYNIER, M.V. \& TENENBAUM, D.R. 1992. Estudo hidrográfico e planctonológico da região entre Cabo Frio (Rio de Janeiro) e o Arquipélago de Abrolhos (Bahia) - Brasil. Nerítica 7 (1-2):71-86.

BÜCKMANN, A. \& KAPP, H. 1975. Taxonomic characters used for the distinction of species of Appendicularia. Mitt. Hamburg. Zool. Mus. Inst. 72:201-228.

CAMPOS, M.A.G. \& VEGA-PÉREZ, L.A. 2002. Oikopleura longicauda (Vogt, 1854) (Chordata: Appendicularia) da região sudeste-sul do Brasil. Simpósio Brasileiro de Oceanografia. CD Rom.

CAMPOS, M.A.G. \& VEGA-PÉREZ, L.A. 2003. Apendiculárias da região entre Cabo Frio e Ilha Grande (RJ) e sua relação com a hidrodinâmica local. In Anais do III Congresso Brasileiro de Pesquisas Ambientais e Saúde, CBPAS. 27 a 30.07.2004. CD-rom, p.28-31.

CAPITANIO, F.L, CURELOVICH, J., TRESGUERRES, M., NEGRI, R.M., VIÑAS, M.D. \& ESNAL, G.B. 2008. Seasonal cycle of appendicularians at a coastal station ( $38^{\circ} 28^{\prime}$ 'S $57^{\circ} 41^{\prime} \mathrm{W}$ ) of the SW Atlantic Ocean. Bull.Mar. Sci. 82:171-184.

CAPITANIO, F.L. \& ESNAL, G.B. 1994. Caracterización morfométrica de los estadios de madurez de Oikopleura fusiformis (Tunicata, Appendicularia). Physis 52(122-123):13-17.

CAPITANIO, F.L. \& ESNAL, G.B. 1997. Appendicularian distribution in the Rio de La Plata estuary and adjacent neritic areas. Neritica 11:37-48.

CAPITANIO, F.L. \& ESNAL, G.B. 1998. Vertical distribution of maturity stages of Oikopleura dioica (Tunicata, Appendicularia) in the frontal system off Valdés Peninsula, Argentina. Bull. Mar. Sci. 6(3):531-539.

CAPITANIO, F.L., MARSCHOFF, E.R. \& ESNAL, G.B. 1995. Distribution and characterization of the maturity stages of Oikopleura dioica (Tunicata, Appendicularia) in the area of Peninsula Valdés, Argentina. Iheringia, Sér. Zool. 79:59-66.

CAPITANIO, F., PÁJARO, M. \& ESNAL, G.B. 2005. Appendicularians: an important food supply for the argentine anchovy. Engraulis anchoita in coastal waters. J. Appl. Ichthyol. 21:414-419. http://dx.doi.org/10.1111/j.1439-0426.2005.00657.x

CASTELLANO-OSÓRIO, L.A. 2003. Appendicularians (Tunicata) of Banco Chinchorro. Caribbean Sea. Bull. Mar. Sci. 73:133-140.

CASTELLANOS, L.A. \& SUÁREZ-MORALES, E. 2009. Appendicularia (Urochordata) of the Gulf of Mexico. In Gulf of Mexico-Origins, waters, and biota. Biodiversity (D.L. Felder \& D.K. Camp, ed.). Texas A\&M Press, College Station, Texas, p.1217-1221.

COTO, F.C. 1965. Contribución al conocimiento de las Apendiculárias del arrecife "La Blanquilla" Veracruz, México, con descripción de una nueva especie. Anales del Centro de Ciências del Mar y Limnologia. UNAM. p.1-20.

DEIBEL, D. 1998. Feeding and metabolism of Appendicularia. In The biology of pelagic tunicates. (Q. Bone, ed.). Oxford University Press, p.139-150.

DEIBEL, D. \& PAFENHÖFFER. 2009. Predictability of patches of neritic salps and doliolids (Tunicata, Thaliacea). J. Plank. Res. 31(12):1571-1579. http:// dx.doi.org/10.1093/plankt/fbp091

DI MAURO, R., CAPITANIO, F \& VIÑAS, M.D. 2009. Capture efficiency for small dominant mesozooplankters (Copepoda, Appendicularia) off Buenos Aires Province $\left(34^{\circ} \mathrm{S}-41^{\circ} \mathrm{S}\right)$. Argentine Sea, using two plankton mesh sizes. Braz. J. Oceanogr. 57(3):205-213. http://dx.doi.org/10.1590/S1679-87592009000300004

EKAU, W. 1999. Topographical and hydrographical impacts on zooplankton community structure in the Abrolhos Bank region, East Brazil. Arch. Fish. Mar. Res. 47(2-3):307-320.

ESNAL, G.B. 1972. Apendicularias de la desembocadura del Rio de La Plata. Physis, Secc. A, 31(82):259-272.

ESNAL, G.B. 1973. Apendicularias de las costas argentinas. Physis, Secc. A 32(85):267-273.

ESNAL, G.B. 1978 (1979). Características generales de la distribucion de tunicados pelagicos del Atlantico Sudoccidental com algunas observaciones morfologicas. Physis, Secc. A 38(94):91-102.

ESNAL, G.B., DAPONTE, M.C. \& SIMONE, L.C. 1993. Pelagic tunicates (Appendicularia and Thaliacea) from the Tropical Western Pacific Ocean. Physis, Secc. A 48(114-115):41-48.

ESSENBERG, C.E. 1926. Copelata from San Diego region. Univ. Calif. Publ. Zool. 28:399-521.

FENAUX, R. 1963. Écologie et biologie des Appendiculaires méditerranéens (Villefranche-Sur-Mer). Vie et Milieu supl. 16:1-142. 
Appendicularia do Estado de São Paulo

FENAUX, R. 1966. Synonymie et distribution géographique des Appendiculaires. Bull. Inst. Océanogr., Monaco 66 (1363):1-23.

FENAUX, R. 1968b. Quelques aspects de la distribution verticale chez les appendiculaires en Méditerranée. Cah. Biol. Mar. 9:23-29.

FENAUX, R. 1976. Cycle vital d'un appendiculaire Oikopleura dioica Fol, 1872 description et chronologie. Annls Inst. océanogr., Paris 52(1):89-101.

FENAUX, R. 1998. The classification of Appendicularia. In The biology of pelagic tunicates (Q. Bone, ed). Oxford University Press, p.251-264.

FENAUX, R. \& GORSKY, G. 1983. Cycle vital et croissance de 1'appendiculaire Oikopleura longicauda (Vogt), 1854. Annls Inst. Océanogr., Paris 59(2):107-116.

FENAUX, R. \& PALAZZOLI, I. 1979. Estimation in situ d'une population d'Oikopleura longicauda (Appendicularia) à l'aide de deux filets de maille différente. Mar. Biol. 55:197-200. http://dx.doi.org/10.1007/BF00396818

GALLIENNE, C.P. \& ROBINS, D.B. 2001. Is Oithona the most important copepod in the world's ocean? J. Plankt. Res. 23(12):1421-1432. http://dx.doi. org/10.1093/plankt/23.12.1421

HANSEN, J.L.S., KIØRBE, T. \& ALLDREDGE, A.L. 1996. Marine snow derived from abandoned larvacean houses: sinking rates, particles content and mechanisms of aggregate formation. Mar. Ecol. Prog. Ser. 141:205-215. http://dx.doi.org/10.3354/meps141205

HIDAKA, K. 2008. Species composition and horizontal distribution of the appendicularian community to the Kuroshio in winter-early spring. J. Plank. Res. 3:152-164

JASPERS, C., NIELSEN, T.G., CARSTENSEN, J., HOPCROFT, R.R. \& MØLLER, E.F. 2009. Metazooplankton distribution accross the Southern Indian Ocean with emphasis on the role of Larvaceans. J. Plank. Res. 31:525-540. http://dx.doi.org/10.1093/plankt/fbp002

KOTT, P. \& ESNAL, G. 2009. Tunicata. In New Zealand inventory of biodiversity (D. Gordon, ed.). Kingdom Animalia, 1:584p.

LENZ, J. 2000. Introduction. In ICES Zooplankton methodology manual. (R.P. Harris, P.H. Wiebe, J. Lenz, H.R. Skjoldal \& M. Huntley, ed.). Academic Press, London.

LÓPEZ-URRUTIA, Á., HARRIS, R. \& SMITH, T. 2004. Predation by calanoids copepods on the appendicularian Oikopleura dioica. Limnol. Oceanogr. 49(1):303-307.

LOPES, R.M., MONTÚ, M.A., GORRI, C.H., MUXAGATA, E., MIYASHITA, L. \& OLIVEIRA, L.P. 2006. O zooplâncton marinho na região entre Cabo de São Tomé (RJ) e o Chui (RS). In O ambiente oceanográfico da Plataforma Continental e do talude na região sudeste-sul do Brasil. (C.L.B. RossiWongtschowski \& L.S-P. Madureira, eds.). p.265-358.

NEUMANN-LEITÃO, S., GUSMÃO, L.M.O., ALMEIDA E SILVA, T., NASCIMENTO-VIEIRA, D.A. \& SILVA, A.P. 1999. Mesozooplankton biomass and diversity in coastal and oceanic waters off North-Eastern Brazil. Arch. Fish. Mar. Res. 47(2-3):153-165.

OHTSUKA, S., NOBUTAKA, K., OKODA, M. \& GUSHIMA, K. 1993. Attachment and feeding of pelagic copepods on larvacean houses. J. Oceanogr. 49(1):115-120. http://dx.doi.org/10.1007/BF02234012

RAZOULS, C., RAZOULS, S. \& SCHUTZE, M.L.M. 1987. Étude quantitativo du mesozooplâncton. In Terres Australis et Antarctiques Françaises. Mission de recherche. Les rapports des campagnes a la mer a bord du "Marion Dufresne" (A. Guill \& J.M., ed.). p.108-116.

SHIGA, N. 1985. Seasonal and vertical distributions of Appendicularia in Volcano Bay, Hokkaido, Japan. Bull. Mar. Sci. 37(2):425-439.

STIBOR, H., VADSTEIN, O., LIPPERT, B., ROEDER, W. \& OLSEN, Y. 2004. Calanoid copepodds and nutrients enrichment determine population dynamics of the appendicularian Oikopleura dioica: a mesocosm experiment. Mar. Ecol. -Prog. Ser. 270:209-215.

THOMPSON, H. 1948. Pelagic tunicates of Australia. Commonwalth Council for Scientific and Industrial Research, Melbourne, 196p.

TOKIOKA, T. 1955. General consideration on japanese appendicularian fauna. Publs. Seto Mar. Biol. Lab. 6(2-3):251-561.

TOKIOKA, T. 1957. Two new Appendicularians from the eastern Pacific, with notes on the morphology of Fritillaria aequatorialis and Tectillaria fertilis. Am. Microsc. Soc. 76(4):359-365. http://dx.doi.org/10.2307/3223769

TOKIOKA, T. 1960. Studies on the distribution of appendicularians and some thaliaceans of the North Pacific, with some morphological notes. Publs. Seto mar. Biol. Lab. 8(2):351-443.

TOMITA, M., SHIGA, N. \& IKEDA, T. 2003. Seasonal occurrence and vertical distribution of appendicularians in Toyama Bay, southern Japan Sea. J. Plankt. Res. 25(6):579-589. http://dx.doi.org/10.1093/plankt/25.6.579

TROEDSSON, C., BOUQUET, J.M., AKSNES, D.G. \& THOMPSON, E.M. 2002. Resource allocation between somatic growth and reproductive output in the pelagic chordate Oikopleura dioica allows opportunistic response to nutritional variation. Mar. Ecol. -Prog. Ser. 243:83-91. http://dx.doi.org/10.3354/ meps 243083

VALENTIN, J.L. 1984. Spatial structure of the zooplankton community in the Cabo Frio region (Brazil) influenced by coastal upwelling. Hydrobiologia 113:183-199. http://dx.doi.org/10.1007/BF00026607

VALENTIN, J.L. 1989. A dinâmica do plâncton na ressurgência de Cabo Frio (RJ). In Memórias do III Encontro Brasileiro de Plâncton (F.P. Brandini, ed.). Curitiba, p.26-35.

VALENTIN, J.L., MONTEIRO-RIBAS, W.M. 1993. Zooplankton community structure on the east-southeast brazilian continental shelf (18-23 $\mathrm{S}$ latitude). Continent. Shelf Res. 13(4):407-424. http://dx.doi.org/10.1016/0278-4343(93)90058-6

VALENTIN, J.L., MONTEIRO-RIBAS, W.M., MUREB, M.A. \& PESSOTTI, E. 1987. Sur quelques zooplanctontes abondants dans l'upwelling de Cabo Frio (Brèsil). J. Plankt. Res. 9(6):1195-1216. http://dx.doi.org/10.1093/plankt/9.6.1195

VANNUCCI, M. \& QUEIROZ, D. 1963. Plâncton coletado durante a VII Viagem do N/E “Almirante Saldanha”: Relatório. Contrções Inst. Oceanogr., Univ. S. Paulo 4:1-9.

VEGA-PÉREZ, L.A. 1993. Estudo do zooplâncton da região de Ubatuba, Estado de São Paulo. Publção Esp. Inst. Oceanogr., S Paulo 10:65-84.

VEGA-PÉREZ, L.A. 2008. Zooplâncton. In Oceanografia de um ecossistema subtropical. Plataforma de São Sebastião, SP. (A.M.S. Pires-Vanin, ed.). EDUSP, São Paulo, p.253-272.

VEGA-PÉREZ, L.A. \& CAMPOS, M.A.G. 2002. Influência das massas de água da região sudeste-sul do Brasil na distribuição de Fritillaria pellucida (Busch, 1851) (Chordata: Appendicularia). Anais do II Congresso Brasileiro de Pesquisas Ambientais e Saúde, CBPAS. 21 a 24.07.2002. CD-rom.

ZUBKOV, M.V. \& LÓPEZ-URRUTIA, A. 2003 Effect of appendicularians and copepods on bacterioplankton composition and growth in the Channel. Aquat. Microb. Ecol. 32:39-46. http://dx.doi.org/10.3354/ame032039 